\title{
MULTIFOCAL DISCHARGES AND ICTAL CORRELATES
}

Continuous EEG/video recordings on 4 children with the interictal EEG pattern of multifocal independent epileptiform discharges (MIED) are reported from the Epilepsy Center, The Johns Hopkins University School of Medicine, Baltimore, MD. The predominant seizure pattern consisted of bilateral tonic seizures with fencing postures. Most seizures arose from surgically resectable regions and epilepsy surgery performed in three patients resulted in improved seizure control. One patient had a focal resection of superior frontal-parietal cortex and two had hemidecorticectomies. Because the scalp recorded EEG data did not provide adequate localization, intracranial electrodes were placed in 2 patients prior to the resections (Burnstine TH et al. Multifocal independent epileptiform discharges in children: ictal correlates and surgical therapy. Neurology August 1991; 41:1223-1228).

COMMENT. The presence of bilateral independent or generalized epileptiform discharges as in patients with MIED is not an absolute contraindication to performing a hemispherectomy, according to the experience of these authors. If the majority of seizures do not originate in one hemisphere and the patient is having frequent generalized seizures, then a corpus callosotomy should be considered.

\section{BENIGN PARTIAL NONROLANDIC EPILEPSIES}

The syndrome of benign partial epilepsy with particular reference to the non-rolandic types is described and the relevant literature reviewed from the Pediatric Seizure Clinic and EEG Laboratory, Beilinson Medical Center, Petah Tikva, and the Sackler Faculty of Medicine, Tel Aviv University, Tel Aviv, Israel. Benign Partial Epilepsy is defined as a primary convulsive disorder belonging within the group of age- and localization-related idiopathic epilepsies and characterized by seizures, predominantly partial and amenable to anticonvulsant therapy, that begin in childhood and remit spontaneously before adulthood. The seizures, usually short and mild, include motor and/or somatosensory symptoms and tend to generalize. They are of variable frequency and occur more frequently in sleep. EEG reveals typical focal features on a normal background that tend to normalize during adolescence. Benign epilepsy occurs in normal children having no neurological or intellectual deficits and no history of brain damage. Benign partial epilepsies other than the rolandic type include occipital epilepsy, benign frontal epilepsy, benign epilepsy with affective symptomatology, benign epilepsy with extreme somatosensory evoked potentials, benign partial epilepsy of adolescence and benign epilepsy associated with multiple spike foci. The Landau-Kleffner syndrome is sometimes included among the benign epilepsies because the seizures cease before the age of 12 and the EEG normalizes before the age of 15 . Aphasia resolves completely or partially in only $50 \%$ of patients. Headache and vomiting or migrainous symptoms are common in benign occipital epilepsy. Children with benign occipital epilepsy associated with headache respond best to phenytoin which relieves both seizures and headaches. (Lerman P, Kivity S. The benign partial nonrolandic epilepsies J Clin Neurophysiol July $1991 ; \underline{8}: 275-285$ ). 
COMMENT. The recognition of the partial epilepsies of childhood is important from the point of view of treatment and prognosis. In children with infrequent seizure occurrence and a diagnosis of benign partial epilepsy the use of potentially toxic anticonvulsant medications may be deferred.

\section{GENETICS OF FEBRILE SEIZURES}

Waking and sleep EEGs were recorded in 67 siblings of 52 patients with febrile seizures (FS) at the Epilepsy Centre Bethel, Bielefeld, F.R.G. Epileptic activity was recorded in at least one sibling of $54 \%$ of patients. Epileptic discharges were noted in $50 \%$ of the 67 siblings; only in waking in $3 \%$, in waking and sleep in $31 \%$ and in sleep only in $15 \%$. The greatest number of epileptic discharges were noted in the waking state during hyperventilation (33\%) and during sleep stage C (39\%). Of nine patients with complicated febrile seizures $56 \%$ had at least one sibling with seizure discharges and of 43 patients with simple febrile seizures $54 \%$ had a sibling with seizure discharges. Of nine patients with later onset epileptic seizures, $44 \%$ had seizure discharges in the EEG. Siblings age six to ten years had the highest rates of activation (67\%). Epileptic discharges occurred in $83 \%$ of siblings with seizures, but in only $46 \%$ of those without seizures. Epileptic activity in patients and in siblings was significantly greater in those who had occipital slow wave activity in the EEG. A multifactorial inheritance of EEG abnormalities was suggested and the genetic factor was important in patients with both simple or complicated FS. Exogenous brain damage may act as an accidental event or catalytic effect leading to the activation of FS, mental retardation, neurologic deficits, behavior disturbances, focal FS, and/or a higher rate of later epileptic seizures (Degen R. et al. A contribution to the genetics of febrile seizures: waking and sleep EEG in siblings. Epilepsia

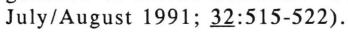

COMMENT. The importance of sleep EEGs and other activating procedures in the prediction of prognosis in patients with febrile seizures is demonstrated by this study. Reports minimizing the value of the EEG in prognosis and later occurrence of epilepsy are often based on EEGs in the waking state only, and without sleep recordings.

A case control study of risk factors for febrile seizures in the People's Republic of China is reported from the Neurological Institute, Sun Yat-Sen University of Medical Sciences, Guangzhou, Beijing Neurosurgical Institute, Beijing, China; National Institutes of Health, Bethesda, MD; and the World Health Organization, Geneva, Switzerland. Information was gathered by door-to-door survey in six major cities in the People's Republic of China (Zhao F et al. Epilepsia July/August 1991; 32:510-514). Family history of febrile seizures, birth and postnatal factors were assessed in 182 subjects and in an equal number of matched controls. The odds ratio for risk of FS in children with a family history of FS in a first-degree relative was 10 . Apart from maternal acute respiratory infection during the first 